\title{
Scaling Capacity by Two Channels in IEEE 802.11 Ad Hoc Networks With an SIR Comparison Algorithm
}

\author{
Ping Chung Ng, Student Member, IEEE, David J. Edwards, and Soung Chang Liew, Senior Member, IEEE
}

\begin{abstract}
When an IEEE 802.11 ad hoc network achieves a capacity $C$ using a single channel, the targeted capacity using two channels should be $2 \cdot C$. We believe that this should be used as the benchmark for capacity comparisons for multichannel schemes. However, most of the dual-channel 802.11 protocols proposed in the literature appear to achieve less than $60 \%$ of the 2 - $C$ targeted capacity. In previous work, we proposed a linkdirectionality-based dual-channel medium-access-control (MAC) protocol (DCP) to boost the network capacities by up to $78 \%$ of our targeted capacities, i.e., $78 \% \times 2 \cdot C=1.56 \cdot C$. However, the DCP still fails to reach the $2 \cdot C$ capacity target due to the overheads incurred by the protocol. In this paper, we implement a signal-to-interference ratio (SIR) comparison algorithm (SCA) on top of the DCP in an attempt to further improve network capacity. This algorithm incurs relatively small overheads and can further relax the protocol constraints that are imposed by the virtual carrier-sensing mechanism. Interestingly, while the capacity of the pure DCP decreases when link lengths are short, the capacity of the pure SCA increases when link lengths are short. The two algorithms compensate for the downside of each other to bring about a more uniform capacity improvement. Simulations show that the proposed scheme [described as DCP with SCA (DCPwSCA)] can increase the network throughputs by $430 \%$ in lattice topologies and $213 \%$ in random topologies.
\end{abstract}

Index Terms-Ad hoc network, capacity, IEEE 802.11, medium-access-control (MAC) protocol, multichannel.

\section{INTRODUCTION}

A WIRELESS ad hoc network is a self-configuring peerto-peer network of mobile nodes connected by wireless links. One of the major problems of a wireless ad hoc network is limited capacity due to its carrier-sensing mechanism. A wireless terminal decides if it should transmit data based on its local environment. The terminal transceiver can sense wireless

Manuscript received December 6, 2008; revised May 16, 2009, August 18, 2009 and December 2, 2009. First published January 19, 2010; current version published May 14, 2010. This work was supported in part by the Competitive Earmarked Research Grant under Project 414507, established under the University Grant Committee of the Hong Kong Special Administrative Region, China, and in part by the Direct Grant under Project 2050436 from the Chinese University of Hong Kong. The review of this paper was coordinated by Dr. H. Jiang.

P. C. Ng is with McKinsey \& Company, Hong Kong (e-mail: chung.ng@ stcatz.oxon.org).

D. J. Edwards is with the Department of Engineering Science, Oxford University, OX13PJ Oxford, U.K. (e-mail: david.edwards@eng.ox.ac.uk).

S. C. Liew is with the Department of Information Engineering, The Chinese University of Hong Kong, Hong Kong, and also with Hong Kong Applied Science and Technology Research Institute, Shatin, Hong Kong (e-mail: soung@ ie.cuhk.edu.hk).

Color versions of one or more of the figures in this paper are available online at http://ieeexplore.ieee.org.

Digital Object Identifier 10.1109/TVT.2010.2040847 transmissions within a range called the carrier-sensing range. It can transmit data when the channel is sensed as "idle"; otherwise, it will defer the transmission until after the currently sensing communications. If two wireless links are packed closely together, simultaneous transmissions will be disallowed by the radio protocol. Therefore, only one transmission is allowed at one time within a carrier-sensing range. This severely limits the network capacity, particularly when the carrier-sensing range is large. There are many capacity enhancement schemes proposed in the literature. One of the approaches is to use an additional radio spectrum resource (multichannel schemes).

When a wireless network uses more channel resources for transmissions, it should achieve a proportionately higher network capacity. If an IEEE 802.11 ad hoc network can achieve capacity $C$ using a single channel, the targeted capacity using $n$ channels should be $n \cdot C$. However, most of the multichannel 802.11 protocols proposed in the literature compared their performance achievements with the original single-channel 802.11 protocol without considering the additional channel resources that they had used.

Our scheme attempts to multiply the network capacity to more than $2 C$ with two channels. It integrates two algorithms: 1) a link-directionality-based dual-channel medium-accesscontrol (MAC) protocol (DCP) and 2) a signal-to-interference ratio (SIR) comparison algorithm (SCA). When combined, these two algorithms compensate for the shortcomings of the other to achieve superior performance in a wide range of situations.

\section{A. Limitation of Our Work}

This paper does not aim to outperform other multichannel protocols in the literature. Instead, we believe that if a singlechannel protocol can achieve a capacity $C$, using $n$ channels should achieve $n \cdot C$. With the proposed dual-channel protocol in this paper, the capacity target is, therefore, $2 \cdot C$. We believe that this capacity target should also be set as the benchmark for fair comparisons between different multichannel algorithms in the field. This paper primarily focuses on single-hop ad hoc networks rather than on multihop environments. Addressing both the single-hop and multihop cases in one single paper will probably be too heavy, and there are many interesting aspects to the single-hop case alone that deserve attention. We believe that the results of this paper can form the foundation for future research for the multihop case.

The preliminary results of this research have been published in [1] and [2]. In [1], $\mathrm{Ng}$ et al. introduced the concept of 
link directionality, whereas [2] proposed the use of a power exchange algorithm to further increase the network throughput. They form the foundation of this paper. After presenting our research results in conferences, we have worked on the feedback and addressed the issues raised. Therefore, the contribution of this paper is twofold.

1) We analyze the capacity of the proposed schemes to understand the major contributor for throughput enhancements under various link-length scenarios.

2) We have revised the details of the SCA and conducted further simulations for lattice and random topologies with various link lengths. This provides further justifications of the throughput improvements achieved by the proposed schemes.

\section{RELATED WORK}

To date, many multichannel protocols for 802.11 ad hoc networks have been proposed. In [3], Mo et al. compared these protocols and classified them into four categories: 1) a dedicated control channel [4], [5], 2) common hopping [6], 3) split phase [7], [8], and 4) multiple rendezvous [9]. Our proposed protocol does not belong to these categories. In fact, it is a novel approach to assign channels to links according to their directionalities.

References [4] and [5] proposed the use of a control channel to exchange request-to-send (RTS)/clear-to-send (CTS) frames, which contain the channel information. Then, nodes use the agreed data channels to send DATA and ACK frames. These protocols require a separate control channel that does not carry data packets. This significantly increases the overhead that is incurred by the protocol. For example, if three channels are used, the targeted capacity would be $3 \cdot C$. One of the three channels, however, is assigned as the control channel, which wastes part of the data transmission capacity.

Another approach is to use frequency hopping [6]. Nodes use preassigned hopping patterns to switch channels for transmitting RTS/CTS frames until agreements are made between nodes. Then, they will use the concurred channels for data transmission. As mentioned in [3], these protocols may incur significant overheads due to the frequent channel switching.

References [7] and [8] proposed to split the transmission time into two phases: 1) control phase and 2) data phase. During the control phase, all nodes switch to the control channel and allocate the transmission channels for the next data phase. These protocols require synchronization between nodes, which is difficult to achieve in distributed ad hoc networks. In addition, during the control phase, no data can be transmitted in other data channels. This, again, wastes the communication resource.

In recent years, there has been further research on multichannel schemes. Many of them [10]-[12] tried to further increase the network capacity by extending the schemes described above or combining these schemes together. In [10], Chen et al. used a split-phase approach with two phases: 1) channel negotiation and 2) data transmissions. The proposed protocol can adjust the size of the negotiation interval according to the observed network traffic condition. This minimizes the airtime used for channel allocations, which, in turn, allows more data trans- missions. In [11], the dedicated control-channel scheme and the split-phase approach are combined. The protocol uses two transceivers to carry out channel negotiations on two channels. It saves half of the time for channel allocations and, thus, leaves more time for data transmissions. In [12], Lin et al. report a dedicated control-channel scheme, which selects a free data channel for each transmission according to the time interval of exchanging the CTS control signal packets. It uses control channel bandwidth more efficiently to reduce the chance of shared channel congestion, particularly in heavily congested networks. In [13], time offsets between channels were introduced for asynchronous multichannel environments. Nodes that have no tasks involved in the current channel can switch to some other channels for more data transmission trials, thereby enhancing the total throughput. Finally, [14] suggested using multiple network interface cards at a node for simultaneous communications. However, the required frequency filtration in transceiver design may be difficult to implement.

Most of the above multichannel 802.11 protocols proposed in the literature (e.g., [4], [6], [7], [13], and [15]) appear to achieve less than the $n \cdot C$ targeted capacity, where $n$ is the number of channels used, and $C$ is the capacity achieved by a single-channel protocol. Their inefficiencies can be attributed to three reasons: 1) A dedicated control channel is used to allocate transmission channels; 2) the overhead that is induced by extra information added to the packet headers; and 3) the transmissions of RTS/DATA and the receptions of CTS/ACK by a node are assigned to the same channel, which limit the potential of simultaneous transmissions (details will be explained in Section III). Compared with the above protocols, our proposed scheme does not require a dedicated control channel and incurs relatively small header overheads. Link-directionality-dependent juxtaposition of control and data information in both channels allows us to increase spatial reuse. In addition, our protocol does not need synchronization between distributed nodes and the channel hopping of the radio transceiver, reducing the protocol overheads.

\section{LINK-DiRECTIONALITY-BASED DUAL-CHANNEL MEDIUM-ACCESS CONTROL PROTOCOL}

Here, we outline the DCP [1]. To avoid simultaneous transmissions that may lead to collisions, the 802.11 protocol uses short RTS and CTS messages to notify other nodes within the virtual carrier-sensing range (VCSRange) to update their network-allocation vectors (NAVs). The NAV includes the duration of the ongoing transmission. Thus, no other nodes within the VCSRange can begin transmission before the NAV expires. Fig. 1 shows an example. Under the 802.11 protocol with the RTS/CTS access mode, none of the links B, C, and D can transmit at the same time with link A. As a result, only one link inside the VCSRange region can transmit at one time. Note that there are two carrier-sensing ranges: 1) the virtual carrier-sensing range (VCSRange) and 2) the physical carriersensing range (PCSRange). For the first one, RTS/CTS can be decoded if the distance of transmission is less than the virtual carrier-sensing range (VCSRange). The NAV that is included in the RTS/CTS frames would prevent nodes from transmitting. 


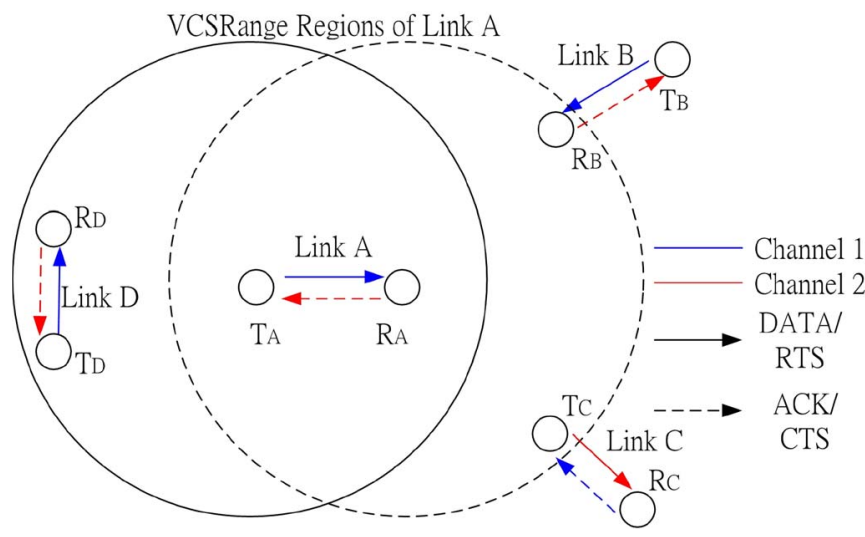

Fig. 1. Network topology with transmission channels assigned by the DCP.

For the second one, physical carrier sensing may also prevent simultaneous transmissions. When the preamble of the PHY header can be decoded, the length field in the PHY header informs the receiver of the duration of the payload that follows. In this paper and in our simulations, we assume that PHY headers are set to be transmitted at the same rate as RTS/CTS frames so that the VCSRange is the same as the PCSRange.

For capacity improvement, we could split the transmissions between the two nodes of a link into two channels based on their directionalities. Let us consider the case where there are two channels, i.e., channels 1 and 2. Links transmit RTS and DATA in one channel (i.e., either both in channel 1 or both in channel 2), and CTS and ACK in the other channel (i.e., either both in channel 1 or both in channel 2). The channels are dynamically assigned based on the directionality, network topology, and who else is transmitting in the neighborhood. Henceforth, by "channel pairing," we refer to the assignment of the channel pair to RTS/DATA and CTS/ACK of the links in the network. With respect to Fig. 1, the main idea is to assign channels to allow collision-free simultaneous transmission of another link $i$ within the VCSRange region of $R_{A}$ and $T_{A}$. Fig. 1 shows a scenario of channel assignment that allows links B and $\mathrm{C}$ to simultaneously transmit with link A. More generally, given link A and another link $i$ with only one of its node within link A's VCSRange, there are two possible cases.

1) The channel pairing is such that the transmissions of link $i$ (link B or C in Fig. 1) within the VCSRange of link A do not affect the reception of RTS/DATA at $R_{A}$ (CTS/ACK at $T_{A}$ ). For example, in Fig. $1, R_{B}$ uses channel 2 for transmission, which does not interfere with the reception at $R_{A}$ in channel 1 .

2) The channel pairing is such that the transmissions of another link $i$ (link B or C in Fig. 1) use the same channel as the reception of $R_{A}\left(T_{A}\right)$, but the transmissions are far enough from $R_{A}\left(T_{A}\right)$ that there is no collision when link $i$ and link A transmit simultaneously. For example, in Fig. 1, $T_{B}$ uses channel 1 for transmission, and $R_{A}$ uses the same channel for reception. Since $T_{B}$ is far away from $R_{A}, R_{A}$ can still successfully receive data from $T_{A}$.

For case 1, we assume that the radio transceiver of wireless nodes can successfully filter signals in one channel from signals in another nonoverlapping channel. For example, in Fig. 1,
$R_{A}$ can extract signals in channel 1 from $T_{A}$, although $R_{B}$ is transmitting with channel 2.

For case 2 , let $d_{T A-R A}$ be the distance between $T_{A}$ and $R_{A}$, and let $d_{T i-R A}$ be the distance between $T_{i}$ and $R_{A}$, and assume that the capture threshold (CPThreshold) is set to be $10 \mathrm{~dB}$. From [16], in a two-ray propagation model, assuming noise is negligible, if the SIR at $R_{A}$ is larger than the CPThreshold, $R_{A}$ can capture the signal from $T_{A}$ when $T_{i}$ is transmitting. That is

$$
\begin{aligned}
\mathrm{SIR}=\left(d_{T i-R A} / d_{T A-R A}\right)^{4}> & \text { CPThreshold } \\
& d_{T i-R A}>1.78^{*} d_{T A-R A}
\end{aligned}
$$

In our simulation, the transmission range (TxRange) is set to be $250 \mathrm{~m}$, while the VCSRange is $550 \mathrm{~m}$ by assigning the data rate for sending the DATA/ACK at $12 \mathrm{Mb} / \mathrm{s}$ (orthogonal frequency-division multiplexing, QPSK) and the basic rate for transmitting RTS/CTS at $2 \mathrm{Mb} / \mathrm{s}$ (direct sequence spread spectrum, differential QPSK). In the worst case, where $T_{A}$ and $R_{A}$ are separated by the maximum transmission range $(250 \mathrm{~m})$, $R_{A}$ can capture the signal from $T_{A}$ if $T_{i}$ is located at more than $1.78 \times 250 \mathrm{~m}=445 \mathrm{~m}$ away from $R_{A}$. Since the VCSRange is set to be $550 \mathrm{~m}$, when $T_{i}$ cannot receive CTS from $R_{A}, T_{i}$ must be far enough so that its signal cannot interfere with the reception of the signal from $T_{A}$ at $R_{A}$. This method of channel assignments according to link directionality was implemented in the DCP in [1]. Due to space limitations, see [1] for details.

\section{Dual-Channel Medium-Access Control Protocol With SignAL-TO-INTERFERENCE RATIO COMPARISON ALGORITHM}

\section{A. Concept}

Section III has outlined the channel-pairing process in the DCP, which assigns transmission channels based on the availability of receptions of RTS and CTS packets. In other words, pairwise simultaneous transmissions are allowed if, for each link, at least one of the two nodes is outside the VCSRanges of the transmitter and receiver nodes of another link (see link A with link B or C in Fig. 1). In certain cases, however, this unnecessarily limits the chances for simultaneous transmissions due to the so-called exposed-node phenomenon. Consider two links again, e.g., link $i$ and link $j$. Deriving from (1), we can define the interference range of link $j$ as

InRange $_{j}=(C P \text { Threshold })^{1 / 4} * d_{T j-R j}=1.78^{*} d_{T j-R j}$

where we assume that CPThreshold $=10 \mathrm{~dB}$ since [17] reported that CPthreshold tends to hold at $10 \mathrm{~dB}$ until $24 \mathrm{Mb} / \mathrm{s}$ in real-life experiments.

Simultaneous transmissions are actually permitted if a node of link $i$ using channel 1 (or 2) for transmissions is at a distance

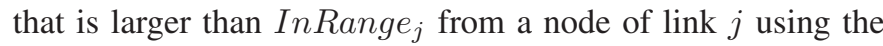
same channel 1 (or 2) for receptions. For example, in Fig. 2, links $\mathrm{A}$ and $\mathrm{B}$ can transmit concurrently, as $T_{B}$ is more than InRange $_{A}$ away from $R_{A}$. From (2), InRange varies with the distance between the two nodes of a link. The closer the distance between $T_{j}$ and $R_{j}\left(d_{T j-R j}\right)$, the smaller the value

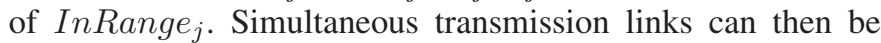




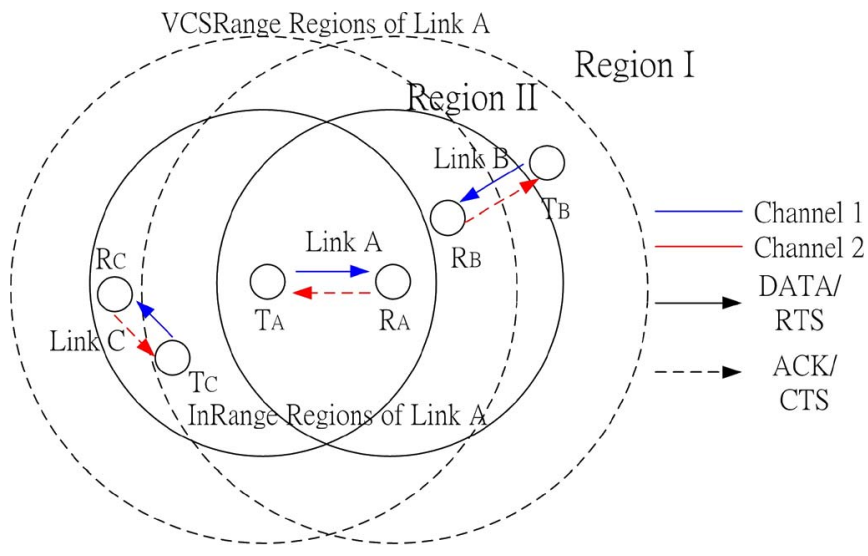

Fig. 2. Closer packing of simultaneous transmissions of links A, B, and C (not link D) is allowed after adopting the SCA in the DCP.

packed closer to each other. This can drastically improve the network capacities, particularly when $d_{T j-R j}$ is small.

Consider Fig. 2 again. Assume that $T_{A}$ uses channel 1 to transmit RTS and DATA to $R_{A}$, whereas $R_{A}$ uses another independent channel 2 to send CTS and ACK back to $T_{A}$. Since $T_{B}$ is outside the interference range of link A (InRange $\left.A\right)$, it can also transmit RTS and DATA via channel 1 . This does not affect the receptions of signals of $R_{A}$ from $T_{A}$ because the signal from $T_{A}$ at $R_{A}$ is more than $10 \mathrm{~dB}$ stronger than the signal from $T_{B}$. In addition, $R_{B}$ uses another independent channel (channel 2) for transmissions that do not interfere with the signal receptions of $R_{A}$. Therefore, links $\mathrm{A}$ and $\mathrm{B}$ can transmit at the same time.

Comparing link B in Figs. 1 and 2, simultaneous transmission links (links A and B) can now be packed closer to each other. This can significantly improve the network capacities. The protocol, however, can no longer assign transmission channels based on the availability of receptions of RTS and CTS packets, as nodes $T_{B}$ and $R_{B}$ can now receive the CTS packets from node $R_{A}$. To solve this problem, we implement an SCA on top of the DCP to identify possible simultaneous transmission opportunities within VCSRanges.

\section{B. $D C P w S C A$}

Here, we describe an SCA for releasing the protocol constraints of the DCP. To realize the concept mentioned in Section IV-A, nodes seek simultaneous transmission opportunities based on the information that is included in the packets received from other links. As shown in Fig. 2, we divide the regions for simultaneous transmissions into two parts.

Region I) A node of link $j$ using channel 1 (or 2) for transmission is more than VCSRange away from a node of link $i$ using channel 1 (or 2) for reception (see Section III).

Region II) A node of link $j$ using channel 1 (or 2) for transmission is more than InRange but less than VCSRange away from a node of link $i$ using channel 1 (or 2) for reception (see Section IV).

For region I, the DCP with an SCA (DCPwSCA) follows the DCP and keeps using the availability of receptions of RTS and
CTS packets to decide channel assignments. See Section III and [1] for details.

For region II, we introduce an SCA to assign channels based on the information that is included in the packets received from other links. The DCPwSCA first utilizes the information from the SCA to look for simultaneous transmission opportunities (for region II).

The algorithm SCA (for region II) always has a higher priority than the algorithm DCP (for region I). When the SCA fails to determine the channels for simultaneous transmissions, the protocol will then fall back to use the DCP. For example, when node $R_{j}$ of link $j$ is within the VCSRange of another link $i$ while node $T_{j}$ is located in region I (outside the VCSRange of another link $i$ ) such that RTS/CTS packets cannot be decoded, $R_{j}$ and $T_{j}$ will then use the SCA and the DCP, respectively, to allocate available channels for simultaneous transmissions.

In this paper and our simulations, we assume that the PHY header is set to be transmitted at the same rate as RTS/CTS frames so that the VCSRange is the same as the PCSRange. In fact, the PCSRange could be larger than the VCSRange in other settings. In those cases, the transmission process of the nodes in region I within the PCSRange of another transmitting node will be halted for sensing the medium activities. However, this could only slightly affect the total capacity performance of our protocol since the airtime for sensing the MAC and PHY headers is relatively small in a complete transmission cycle.

To assign channels for simultaneous transmissions in regions I and II, the DCPwSCA requires two algorithms: 1) the SCA and 2) randomly chosen transmission channels. Algorithm 2 is inherited and modified from the DCP [1].

1) SCA: The main purpose of the SCA is to assign channels for nodes located within VCSRanges but outside InRanges of nodes of other links. The algorithm inserts the signal strength and interference level information of the received packet (RTS/CTS) that is obtained from the measurements of wireless transceivers in the MAC header of the replied packets (CTS/ DATA). Nearby nodes can then extract the signal strength and interference level information that is attached with the MAC header to decide their corresponding transmission channels.

Consider a pair of links, e.g., link $i$ and link $j$, with one of their nodes within region II of the other link. When $R_{i}\left(T_{i}\right)$ of link $i$ receives an RTS (CTS) packet from $T_{i}\left(R_{i}\right)$, it records the signal strength $S_{T i->R i}\left(S_{R i->T i}\right)$ and the interference level $I_{T i->R i}\left(I_{R i->T i}\right)$ of the packet received and adds an additional header in its MAC header of the replied CTS (DATA) packet. Notice that, here, the conditions within the brackets in this subsession represent the scenario when transmitter $T_{i}$ and receiver $R_{i}$ of link $i$ swap their roles during the reception of ACK/CTS, where $T_{i}$ becomes the receiver and $R_{i}$ becomes the transmitter. When other nodes (e.g., $T_{j}$ ) receive the CTS (DATA) packet, they will extract the signal strength $S_{T i->R i}\left(S_{R i->T i}\right)$ and the interference level $I_{T i->R i}\left(I_{R i->T i}\right)$ information from the MAC header. In addition, they will record the receive power Power $_{R i->T j}$ $\left(\right.$ Power $\left._{T i->T j}\right)$ of the CTS (DATA) packet received. Owing to the reciprocal nature of the wireless radio link, we can state that $\operatorname{Power}_{T j->R i} \approx \operatorname{Power}_{R i->T j}\left(\right.$ Power $_{T->T i} \approx$ Power $_{T i->T j}$ ). The algorithm then compares $\operatorname{Power}_{T j->R i}$ 
$\left(\right.$ Power $\left._{T j->T i}\right)$ with $S_{T i->R i}\left(S_{R i->T i}\right)$ and $I_{T i->R i}\left(I_{R i->T i}\right)$. If $\quad S_{T i->R i} /\left(I_{T i->R i}+\right.$ Power $\left._{T j->R i}\right)>C P T h r e s h o l d$ $\left(S_{R i-T i} /\left(I_{R i-T i}+\right.\right.$ Power $\left.\left._{T j->T i}\right)>C P T h r e s h o l d\right)$, simultaneous transmissions are allowed, and the protocol will then assign the same transmission channel to $T_{j}$. Fig. 2 shows an example. $T_{B}$ and $R_{B}$, which are located in region II of $R_{A}$ and $T_{A}$, respectively, use the SCA to identify the channels for simultaneous transmissions. Otherwise, when both nodes of link $i$ are within the InRange of link $j$, link $i$ will wait for the expiration of the NAV and then resume the transmission process. For example, in Fig. 2, links A and $\mathrm{C}$ will take turn to transmit since both $T_{C}$ and $R_{C}$ are within the InRange of $T_{A}$. In addition, each node records the assignment result of the SCA in a channel-assignment table.

2) Randomly Assigned Transmission Channels: When a transmitter of a link is allowed by the protocol to choose either one of the two channels for simultaneous transmissions, it will randomly pick one of the two channels to send RTS to the receiver of the link. If it can receive CTS that is replied from the receiver, it will keep using that channel for forthcoming transmissions. Otherwise, it will swap to another channel for retransmissions. This algorithm allows the transmitter to identify the suitable transmission channel for the receiver.

\section{Analysis of CAPACITy Improvement}

Here, we analyze the capacity enhancements of the DCP, the SCA, and our proposed protocol (the DCPwSCA). We first calculate the upper capacity bounds of the proposed DCPwSCA, the DCP, and the SCA. Then, we consider the maximum achievable capacity of an ad hoc network using the original 802.11 protocol in the same given network area. Finally, we compare their capacity improvements. Given a network in a large area $S$, the upper capacity bound is the maximum number of simultaneous transmission links that can be packed in area $S$.

Note that, here, the analysis does not calculate the exact network performance since the actual network capacity depends on the given network topology and the traffic condition. See [18], which proposed the use of link-directional interference graphs to evaluate throughput improvements achieved by assigning channels according to link directionalities. Here, instead, we use the upper bound (best-case) analysis to compare the performance of the DCP and the SCA under various link-length scenarios to eliminate the impact of topology and traffic pattern. The analysis justifies that these two algorithms can compensate for the downside one another to bring about a more uniform capacity improvement.

\section{A. Upper Capacity Bound of the DCPwSCA}

To calculate the upper bound on the throughput capacity, the key is to identify the exclusion region that is consumed by each transmission link. The seminal work of Gupta and Kumar [19] and Agarwal and Gupta [20] defined the exclusion region as an area that is associated with an active receiver that must remain disjointed from the exclusion region of every other active receiver in the network. In other words, an exclusion region [21] is a region around each receiver such that no

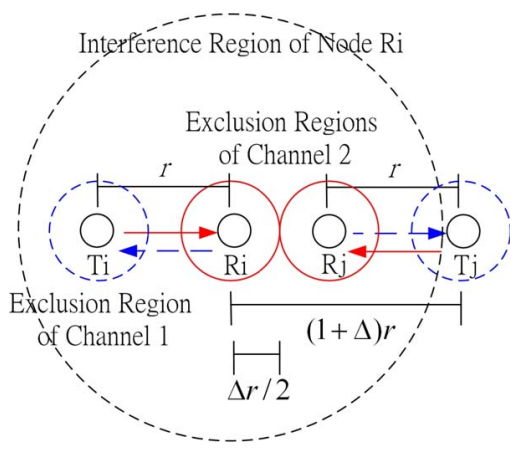

Fig. 3. Exclusion regions of a pair of simultaneous-transmission links using the DCPwSCA.

interferer/transmitter exists inside this region. We apply the concept of the exclusion region to bound the capacities of networks using the original 802.11, DCP, SCA, and DCPwSCA protocols. In our proposed protocol (the DCPwSCA), link $i$ can successfully transmit if one of the two nodes of link $j$ is outside the interference range (InRange) of link $i$. As defined in [19], the interference range of link I

$$
\text { InRange }_{i}=(1+\Delta) r_{i}
$$

where $r_{i}$ is the transmitter-receiver distance of a link $i$, and $\Delta>0$ is related to a power margin. Fig. 3 shows an example when links $i$ and $j$ are packed with the closest distance. Since node $T_{i}\left(T_{j}\right)$ of link $i(j)$ is outside the InRange of $R_{j}\left(R_{i}\right)$, links $i$ and $j$ can transmit concurrently. The distance between $R_{i}$ and $R_{j}$ is equal to $\Delta r$. Thus, the exclusion region of each channel of a link is a circular disk of radius $\Delta r / 2$ that is centered at the receiver of each channel of the link. For simplicity, we consider a network of area $S$ with all links having the same transmitter-receiver length $r$, and the total data rate by using all channels is $W$. The area of the exclusion region of each channel of a link using the DCPwSCA, i.e., $E_{\mathrm{DCPwSCA}}$, is given by

$$
E_{\mathrm{DCPwSCA}}=\frac{\pi \Delta^{2} r^{2}}{4} .
$$

Due to the bidirectional traffic of each link, the upper bound of the throughput capacity per channel then becomes

$$
C_{\mathrm{DCPwSCA}}=\frac{W}{2} \cdot \frac{S}{E_{\mathrm{DCPwSCA}}}=W \frac{2 S}{\pi \Delta^{2} r^{2}} .
$$

Thus, $C_{\mathrm{DCP} w \mathrm{SCA}}$ only depends on the transmitter-receiver distance of a link $(r)$ and is independent of the VCSRange $(v)$.

\section{B. Upper Capacity Bound of the DCP}

In the DCP, simultaneous transmissions are allowed if one of the two nodes of link $j$ is outside the VCSRange of either the transmitter or the receiver of link $i$. Fig. 4 shows an example. Since node $T_{i}\left(T_{j}\right)$ of link $i(j)$ is outside the VCSRange of $R_{j}\left(R_{i}\right)$, links $i$ and $j$ can transmit at the same time. When links $i$ and $j$ are packed with the closest distance, the distance between $R_{i}$ and $R_{j}$ becomes $v-r$. Since the transmitter and the receiver of a link use different channels for receptions, the exclusion region of each channel of a link is a circular disk of 


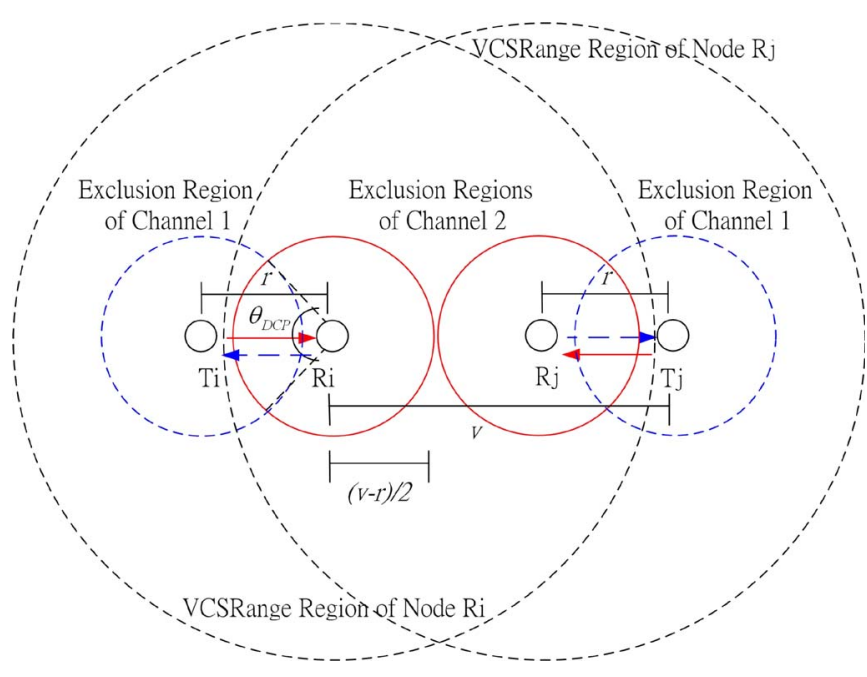

Fig. 4. Exclusion regions of a pair of simultaneous-transmission links using the DCP.

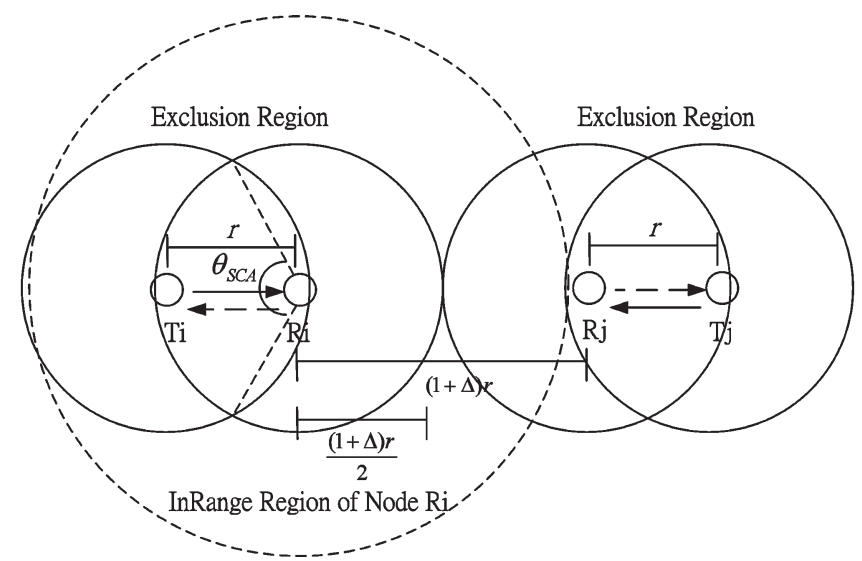

Fig. 5. Exclusion regions of a pair of simultaneous-transmission links using the SCA only (without the DCP).

radius $v-r / 2$ that is centered at the receiver of each channel of the link. The area of the exclusion region of each channel of a link using the DCP, i.e., $E_{\mathrm{DCP}}$, is given by

$$
E_{\mathrm{DCP}}=\frac{\pi(v-r)^{2}}{4} .
$$

The DCP uses two channels for the bidirectional traffic: RTS/DATA in one channel and CTS/ACK in another. For fair comparisons, the upper bound of the throughput capacity per channel then becomes

$$
C_{\mathrm{DCP}}=\frac{W}{2} \cdot \frac{S}{E_{\mathrm{DCP}}}=W \frac{2 S}{\pi(v-r)^{2}} .
$$

As a result, $C_{\mathrm{DCP}}$ can then be expressed in terms of $v$ and $r$.

\section{Upper Capacity Bound of the SCA Only (Without the DCP)}

For comparing the capacity enhancements that are obtained by splitting channels based on link directionalities (DCP) and adopting the SCA in the protocol, here, we also derive the exclusion region of a single-channel protocol with the SCA (without the DCP). Since only one channel is used by the protocol, simultaneous transmissions of links $i$ and $j$ are permitted only if $R_{i}$ and $T_{i}$ are outside the interference range InRange

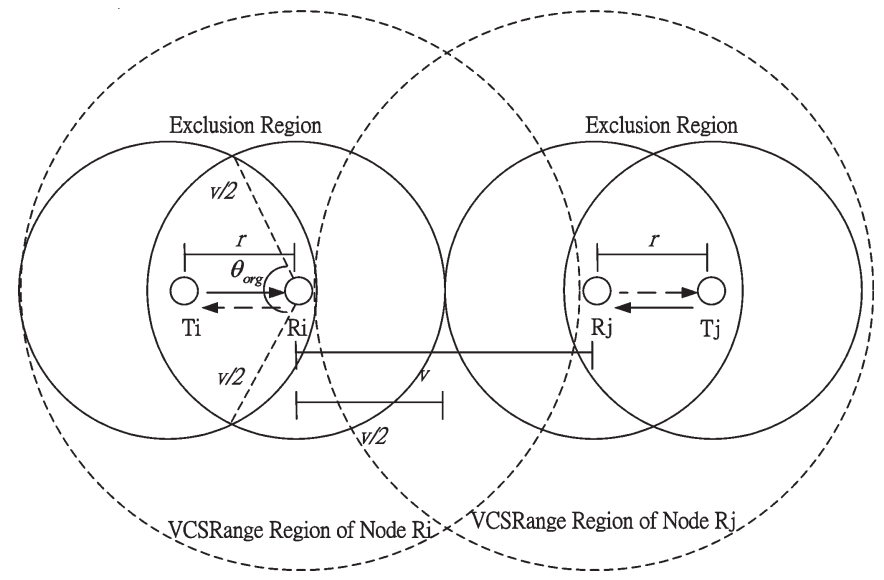

Fig. 6. Exclusion regions of a pair of simultaneous-transmission links using the original 802.11 protocol.

of $R_{j}$ and $T_{j}$, as shown in Fig. 5. Thus, the exclusion region of each link becomes two disks of radius $(1+\Delta) r / 2$ centered at two nodes of the link. To calculate the area of the exclusion region of a link, we first derive the angle $\theta_{\mathrm{SCA}}$, as shown in Fig. 5. Following the similar analysis as shown in [22], we have

$$
\begin{aligned}
\cos \left(\frac{\theta_{\mathrm{SCA}}}{2}\right) & =\frac{r}{2(1+\Delta) r / 2}=\frac{1}{1+\Delta} \\
\theta_{\mathrm{SCA}} & =2 \cos ^{-1}\left(\frac{1}{1+\Delta}\right) .
\end{aligned}
$$

The area of the exclusion region of a link using the SCA, i.e., $E_{\mathrm{SCA}}$, is given by

$$
\begin{aligned}
E_{\mathrm{SCA}} & =\frac{\pi(1+\Delta)^{2} r^{2}}{2} \\
& -\left(\frac{(1+\Delta)^{2} r^{2}}{4} \theta_{\mathrm{SCA}}-r \cdot \frac{(1+\Delta) r}{2} \cdot \sin \frac{\theta_{\mathrm{SCA}}}{2}\right)
\end{aligned}
$$

and the upper bound of the capacity then becomes

$$
C_{\mathrm{SCA}}=W \frac{S}{E_{\mathrm{SCA}}} .
$$

As a result, $C_{\mathrm{SCA}}$ can then be expressed in terms of $r$ and is independent of $v$.

\section{Upper Capacity Bound of the Original 802.11 Protocol}

To calculate the upper capacity bound of the network using the original 802.11 protocol, the key, again, is to identify the exclusion region that is consumed by each link. Fig. 6 shows a pair of links that can transmit simultaneously. Nodes $R_{i}$ and $R_{j}$ of links $i$ and $j$, respectively, are located just outside the VCSRanges of the other link. $R_{i}$ and $R_{j}$ cannot sense the signals from each other, and thus, they can transmit at the same time. In the original 802.11 protocol, $R_{i}$ and $R_{j}$ have to reply ACKs back to the transmitters $T_{i}$ and $T_{j}$. When ACK is transmitting, $R_{i}\left(R_{j}\right)$ becomes a transmitter, while $T_{i}\left(T_{j}\right)$ becomes a receiver. This bidirectional traffic of the original 802.11 protocol further enlarges the exclusion region that is consumed by each link. As shown in Fig. 6, we can define the exclusion region as two disks of radius $v / 2$ centered at two 
nodes of a link, where $v$ is the virtual carrier-sensing range (VCSRange). Once the exclusion regions of links are disjointed, simultaneous transmissions are permitted by the protocol. To calculate the area of the exclusion region of a link, we first derive the angle $\theta_{\text {org }}$, as shown in Fig. 6, in terms of $r$ and $v$. Using the similar approach as (8), we have

$$
\begin{aligned}
\cos \left(\frac{\theta_{\text {org }}}{2}\right) & =\frac{r}{2(v / 2)}=\frac{r}{v} \\
\theta_{\text {org }} & =2 \cos ^{-1}\left(\frac{r}{v}\right) .
\end{aligned}
$$

Thus, the area of the exclusion region of a link using the original 802.11 protocol, i.e., $E_{\text {org }}$, is given by

$$
E_{\text {org }}=\frac{\pi v^{2}}{2}-\left(\frac{v^{2}}{4} \theta_{\text {org }}-r \cdot \frac{v}{2} \cdot \sin \frac{\theta_{\text {org }}}{2}\right) .
$$

The upper bound of the throughput capacity then becomes

$$
C_{\text {org }}=W \frac{S}{E_{\text {org }}} \text {. }
$$

Thus, $C_{\text {org }}$ depends on the VCSRange $v$ and the transmitterreceiver distance $r$. In Section V-E, we will use $C_{\text {org }}$ to compare the capacity improvements that are obtained by the DCP, SCA, and DCPwSCA protocols.

\section{E. Comparisons}

In summary, the areas of the exclusion region of a link using the original 802.11 protocol and the DCP depend on the VCSRange $v$ and the transmitter-receiver distance $r$, whereas those links using the proposed protocol DCPwSCA and the SCA are determined only by $r$ and are independent of $v$. We can express the capacity improvement of the DCPwSCA by comparing with the original 802.11 protocol. That is

$$
I_{\mathrm{DCPwSCA}}(r)=\frac{C_{\mathrm{DCPwSCA}}(r)}{C_{\mathrm{org}}(r)}=\frac{2 \cdot E_{\mathrm{org}}}{\pi \Delta^{2} r^{2}} .
$$

We vary the constant link length assumed in the network from $r_{\max }$ to $r_{\min }$. The average capacity improvement is

$$
I_{\mathrm{DCPwSCA}}=\frac{1}{r_{-} \max -r_{-} \min } \int_{r_{-} \min }^{r_{-} \max } \frac{2 \cdot E_{\text {org }}(r)}{\pi \Delta^{2} r^{2}} d r .
$$

Let us say that the maximum transmitter-receiver distance $r_{\max }$ is $250 \mathrm{~m}$ and that the minimum $r_{\min }$ is $150 \mathrm{~m}$. Then, the average capacity improvement $I_{\mathrm{DCPwSCA}}$ is equal to $440 \%$. This significantly multiplies the network capacities by using two channels. The capacity improvements are obtained by two factors:

1) permitting closer packing of simultaneous transmissions by splitting the reception channels of nodes of each link (by the DCP);

2) releasing the protocol constraints that are induced by the virtual carrier-sensing mechanism (by the SCA).

For factor 1, the DCPwSCA separates the transmission and reception channels of a node. This allows nodes using the same

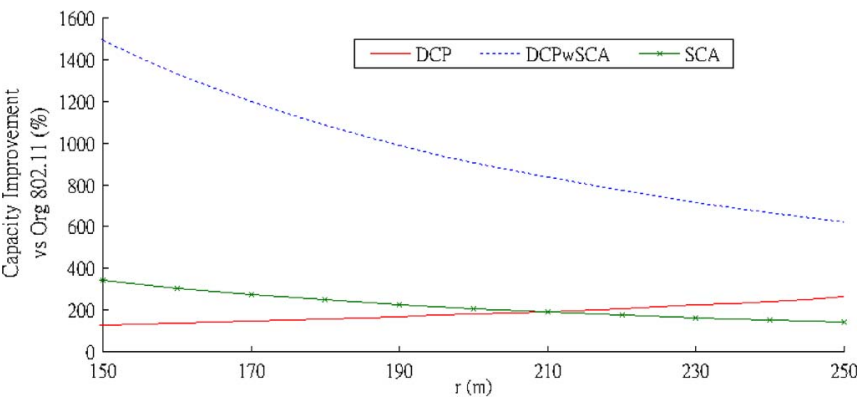

Fig. 7. Theoretical capacity improvements of protocols against the original 802.11 protocol versus the transmitter-receiver distance $r$.

reception channel to be packed closer to each other since their transmissions use another independent channel that does not interfere with the receptions of the other link. For factor 2, both the 802.11 and the DCP relay on their carrier-sensing conditions to justify their transmission processes. The DCPwSCA, on the other hand, bases on its SCA instead of the virtual carrier-sensing mechanism to seek simultaneous transmission opportunities. This, again, allows simultaneous transmissions to be packed closer to each other with interlink distances that are less than the VCSRange.

Fig. 7 plots the capacity improvements that are obtained by the DCPwSCA $\left(I_{\mathrm{DCP} w S C A}(r)\right)$, the DCP $\left(I_{\mathrm{DCP}}(r)\right)$, and the SCA $\left(I_{\mathrm{SCA}}(r)\right)$ when comparing with the original 802.11 protocol versus transmitter-receiver distances $r$. The curves of the DCP and the SCA intercept when $r=210$. When $r \geq 210$, the benefit of the DCP (factor 1) plays a more important role than that of the SCA (factor 2) in terms of capacity improvement. When $r$ increases, the advantage of the SCA diminishes since the size of the InRange of a link expenses and gets closer to the size of the VCSRange. This reduces the benefit obtained by releasing the protocol constraints that are induced by the virtual carrier-sensing mechanism. On the other hand, when $r$ increases, the area of the exclusion region $E_{\mathrm{DCP}}$ decreases [as shown in (7)], and thus, links can be packed closer to each other. This reduces interlink distances and makes factor 1 the dominant factor for capacity improvements.

When $r \leq 210$, the area of the exclusion region, on the other hand, increases when the transmitter-receiver distance $r$ decreases, and thus, the advantage of factor 1 diminishes. However, since the InRange decreases with $r$ [see (9)], factor 2 overrides factor 1 by permitting closer link packing and becomes the prominent factor for capacity improvements.

The DCPwSCA integrates the benefits of the DCP (factor 1) and the SCA (factor 2) in long-range $(r \geq 210)$ and short-range $(r \leq 210)$ transmitter-receiver distance $r$. This significantly reduces the exclusion region $E_{\mathrm{DCPwSCA}}$ and boosts capacities. Theoretically, Fig. 7 shows that the capacity can be boosted from 6.2 to 14.9 times of that of the original 802.11 protocol when $r$ is between 150 and $250 \mathrm{~m}$. In Section VI, we will show the capacity improvements that are obtained by simulations in lattice and random network topologies.

\section{Simulation Results}

We have implemented our proposed MAC protocol (the DCPwSCA) in the NS-2 [23] simulator. We assume that the 


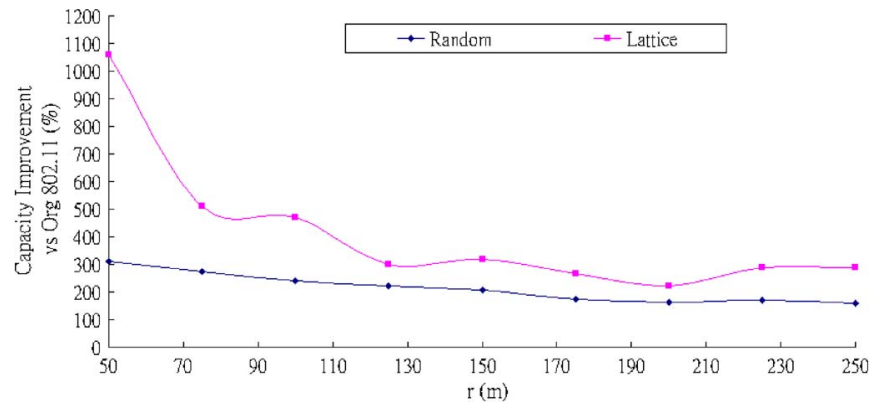

Fig. 8. Simulation results of capacity improvements of the DCPwSCA against the original 802.11 protocol versus the transmitter-receiver distance $r$ in lattice topologies and random topologies with 90 single-hop links.

VCSRange and the TxRange are set to be 550 and $250 \mathrm{~m}$, respectively. All data sources are saturated user datagram protocol traffic streams with a fixed packet size of 1460 B. Note that, here, the original 802.11 protocol with RTS/CTS mechanism is used to compare with the simulation results. When a non-RTS/CTS scheme is used for comparisons, the capacity improvement will be reduced, particularly when the packet size is small.

\section{A. Lattice Topologies}

Fig. 8 shows the capacity improvements that are obtained by the DCPwSCA in lattice topologies. Single-hop links with transmitter-receiver distance $r$ are packed as many as possible in a $2000 \times 2000 \mathrm{~m}$ square. The interlink distance is set to be $2.6 * r$. When $r=50$, the DCPwSCA drastically improves the network capacity of the original 802.11 protocol by 10.59 times. In the worst case, when $r=200$, the improvement is $223 \%$. On average, the capacities are multiplied by $430 \%$. Note that here, the capacity improvement of lattice topology is not comparable with the theoretical results of Fig. 7 since they are based on different topology assumptions. Fig. 7 assumes a perfect topology that allows nodes to fully fill a given area without overlapping or waste of exclusion regions, whereas here, there are areas that are not covered by the exclusion regions of nodes in the lattice topologies.

\section{B. Random Topologies}

In each random network, 90 single-hop links with transmitter-receiver distance $r$ are randomly placed inside a $3000 \times 3000 \mathrm{~m}$ square. The entire topology of each link-length simulation is randomly regenerated. On average, the capacities are boosted by $213 \%$. The lowest improvement is $158 \%$ when $r=250$, and the highest is $310 \%$ when $r=50$.

Simulations show that the DCPwSCA can significantly multiply the network capacities in both lattice and random topologies. We believe that the protocol can outperform other dual-channel schemes (e.g., [15]) that have previously been proposed in the literature.

\section{Multihop Topologies}

We generate a lattice topology of three seven-hop chains. The nodes in a chain are separated by $250 \mathrm{~m}$, while the interchain distance is $500 \mathrm{~m}$. Simulations show that the total throughput of the DCPwSCA is $3.90 \mathrm{Mb} / \mathrm{s}$, whereas that of the original 802.11 protocol is $1.87 \mathrm{Mb} / \mathrm{s}$. The capacity is improved by $109 \%$.

\section{CONCLUSION}

This paper has been an attempt to multiply network capacities of IEEE 802.11 ad hoc networks by two channels. Our scheme (the DCPwSCA) integrates two algorithms to boost network throughputs: 1) a link-directionality-based DCP and 2) an SCA.

For algorithm 1, it splits the transmission and reception channels of a node so that transmissions in one channel do not interfere with the receptions in another nonoverlapping channel. We have shown that this allows more compact packing of nodes using the same reception channel.

For algorithm 2, it releases the protocol constraints that are imposed by the virtual carrier-sensing mechanism. Links can then decide their transmission processes based on the physical interference constraints instead of the receptions of RTS/CTS packets, as in the original 802.11 protocol.

We have studied the performance improvements achieved by algorithms 1 and 2. We have demonstrated that algorithm 1 outperforms algorithm 2 in long intralink distances, whereas algorithm 2 outweighs algorithm 1 in short intralink distances. Interestingly, integrating these two algorithms allows the protocol to compensate for the downsides of one another. Simulations have shown that the DCPwSCA can multiply the network capacities of single-channel IEEE 802.11 ad hoc networks by $430 \%$ in lattice and $213 \%$ in random topologies. We believe that other performance-enhancement schemes (e.g., adaptive power control [24], optimal load control algorithm [25], or directional antenna [26]) can also be adopted with our scheme to further increase network throughputs.

\section{REFERENCES}

[1] P. C. Ng, D. J. Edwards, and S. C. Liew, "Assigning channels by link directionality in a medium access control protocol for IEEE 802.11 ad hoc networks," IET Commun., vol. 3, no. 11, pp. 1736-1746, Nov. 2009.

[2] P. C. Ng, D. J. Edwards, and S. C. Liew, "A link-directionality-based dual channel MAC protocol with a power exchange algorithm for IEEE 802.11 ad-hoc networks," in Proc. IEEE PIMRC, Helsinki, Finland, Sep. 2006, pp. 1-5.

[3] J. Mo, H. W. So, and J. Walrand, "Comparison of multi-channel MAC protocols," IEEE Trans. Mobile Comput., vol. 7, no. 1, pp. 50-65, Jan. 2008.

[4] S. Wu, C.-Y. Lin, Y.-C. Tseng, and J.-L. Sheu, "A new multi-channel MAC protocol with on demand channel assignment for multi-hop mobile ad-hoc networks," in Proc. I-SPAN, Dec. 2000, pp. 232-237.

[5] Y. Tseng, "A multi-channel MAC protocol with power control for multihop mobile ad-hoc networks," in Proc. IEEE Distrib. Comput. Syst. Workshop, Apr. 2001, pp. 419-424.

[6] J. Chen, S.-T. Sheu, and C.-A. Yang, "A new multichannel access protocol for IEEE 802.11 Ad Hoc wireless LANs," in Proc. IEEE PIMRC, Sep. 2003, pp. 2291-2296.

[7] J. So and N. Vaidya, "Multi-channel MAC for ad hoc networks: Handling multi-channel hidden terminals using a single transceiver," in Proc. ACM MobiHoc, May 2004, pp. 222-223.

[8] A. Tzamaloukas and J. J. Garcia-Luna-Aceves, "A receiver-initiated collision-avoidance protocol for multi-channel networks," in Proc. IEEE INFOCOM, Apr. 2001, pp. 189-198.

[9] P. Bahl, R. Chandra, and J. Dunagan, "SSCH: Slotted seeded channel hopping for capacity improvement in IEEE 802.11 ad-hoc wireless networks," in Proc. ACM MobiCom, Sep. 2004, pp. 216-230. 
[10] W. Chen, T.-K. Huang, Y.-C. Chang, and J.-C. Liu, "An adaptive multichannel MAC protocol for wireless ad-hoc networks," in Proc. IEEE ICC, Istanbul, Turkey, 2006, vol. 8, pp. 3651-3656.

[11] S. Lo and C. Tseng, "A novel multi-channel MAC protocol for wireless ad-hoc networks," in Proc. IEEE VTC-Spring, Dublin, Ireland, Apr. 2007, pp. 46-50.

[12] C. Lin, M.-C. Wueng, T.-H. Chiu, and S.-I. Hwang, "Concurrent multichannel transmission (CMCT) MAC protocol in wireless mobile ad-hoc networks," in Proc. IEEE ICACT, Phoenix Park, Korea, Feb. 2007, vol. 1, pp. 445-449.

[13] C. Son, N.-H. Lee, B. Kim, and S. Bahk, "MAC protocol using asynchronous multi-channels in ad-hoc networks," in Proc. IEEE WCNC, Hong Kong, Mar. 2007, pp. 401-405.

[14] C. Xu, K. Liu, Y. Yuan, and G. Liu, "A novel multi-channel based framework for wireless IEEE 802.11 ad-hoc networks," in Proc. IEEE WCNM, Wuhan, China, Sep. 2005, vol. 2, pp. 812-815.

[15] H. Zhai, J. Wang, and Y. Fang, "DUCHA: A new dual-channel MAC protocol for multihop ad hoc networks," IEEE Trans. Wireless Commun., vol. 5, no. 11, pp. 3224-3233, Nov. 2006.

[16] T. Rappaport, Wireless Communications: Principles and Practice. Englewood Cliffs, NJ: Prentice-Hall, 2002.

[17] J. Lee, W. Kim, S.-J. Lee, D. Jo, J. Ryu, T. Kwon, and Y. Choi, "An experimental study on the capture effect in 802.11a networks," in Proc. ACM WiNTECH, Sep. 2007, pp. 19-26.

[18] P. C. Ng, D. J. Edwards, and S. C. Liew, "Colouring link-directional interference graphs in wireless ad hoc networks," in Proc. IEEE GLOBECOM, Washington, D.C., Nov. 2007, pp. 859-863.

[19] P. Gupta and P. R. Kumar, "The capacity of wireless networks," IEEE Trans. Inf. Theory, vol. 46, no. 2, pp. 388-404, Mar. 2000.

[20] A. Agarwal and P. R. Kumar, "Improved capacity bounds for wireless networks," Wireless Commun. Mobile Comput., vol. 4, no. 3, pp. 251261, May 2004.

[21] R. Menon, R. M. Buehrer, and J. H. Reed, "Impact of exclusion region and spreading in spectrum-sharing ad hoc networks," in Proc. ACM TAPAS, Boston, MA, Aug. 5, 2006.

[22] K. Xu, M. Gerla, and S. Bae, "How effective is the IEEE $802.11 \mathrm{RTS} / \mathrm{CTS}$ handshake in ad hoc networks?" in Proc. IEEE GLOBECOM, Nov. 2002, vol. 1 , pp. 17-21.

[23] The Network Simulator-ns2. [Online]. Available: http://www.isi.edu/ nsnam/ns

[24] W. H. Ho and S. C. Liew, "Achieving scalable capacity in wireless networks with adaptive power control," in Proc. IEEE LCN, Nov. 2005, pp. 720-728.

[25] P. C. Ng and S. C. Liew, "Throughput analysis of IEEE 802.11 multi-hop ad hoc networks," IEEE/ACM Trans. Netw., vol. 15, no. 2, pp. 309-322, Apr. 2006.

[26] C. Y. Chang, H.-C. Sun, and C.-C. Hsieh, "MCDA: An efficient multi-channel MAC protocol for 802.11 wireless LAN with directional antenna," in Proc. IEEE AINA, Mar. 2005, pp. 64-67.

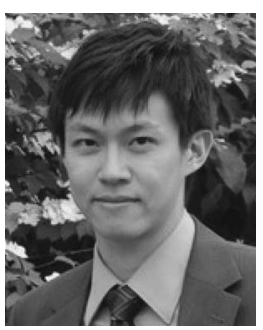

Ping Chung Ng (S'04) received the B.Eng. (highest honor) and M.Phil. degrees in information engineering from The Chinese University of Hong Kong (CUHK), Hong Kong, in 2003 and 2005, respectively, and the D.Phil. (Ph.D.) degree in engineering science from Oxford University, Oxford, U.K., in 2008. He held the Croucher Foundation Scholarship, The Institution of Engineering and Technology Robinson Research Scholarship, and Overseas Graduate Scholarship of St. Catherine's College, Oxford, to work toward the Ph.D. degree.

$\mathrm{He}$ is currently a Management Consultant with McKinsey \& Company, Hong Kong, providing strategic advice to multinational corporations. He has three pending U.S. patents. His current research interests include intelligent transport systems, wireless ad hoc networks, medium-access control, and wireless routing protocols.

Dr. Ng's Master's degree thesis received the Outstanding Thesis Award from the Faculty of Engineering, CUHK. He was also a recipient of the Best Paper Awards at the First IEEE International Conference on Mobile Ad Hoc and Sensor Systems and the Fourth IEEE International Workshop on Wireless Local Network (2004).

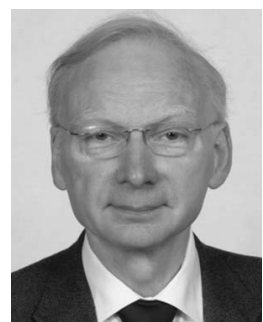

David J. Edwards received the B.Sc., M.Sc., and $\mathrm{Ph} . \mathrm{D}$. degrees from Bristol University, Bristol, U.K.

He has been involved in academics for 24 years (20 years at Oxford University, Oxford, U.K., and four years at Bristol University) after having spent 12 years in the industry with British Telecom. He has authored or coauthored approximately 400 publications in his time as an academic. His research has been funded by many bodies over the years, including research councils, industry, and government agencies. He acts as a consultant to industry and outside bodies. He collaborates widely within Oxford, the U.K., and internationally. His research activities have been in communications systems, radio imaging, medical imaging and electromagnetic measurements, and superconducting devices.

Dr. Edwards is a Chartered Engineer, a Fellow of The Institution of Engineering and Technology, and a Fellow of the Royal Astronomical Society. He has received the following prizes and awards: the 1986 Institution of Electrical Engineers (IEE) Prize for Innovation; the 1990 National Physical Laboratory Metrology Award; the 1990 IEE Mountbatten premium (two papers); the 1990 IEEE Neal Shepherd Memorial Award; and the 2006 Automated RF and Microwave Measurement Society Award.

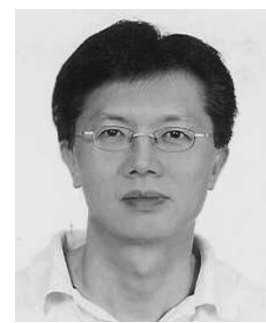

Soung Chang Liew (S'84-M'87-SM'92) received the S.B., S.M., E.E., and Ph.D. degrees from the Massachusetts Institute of Technology (MIT), Cambridge.

From 1984 to 1988 , he was with the MIT Laboratory for Information and Decision Systems, where he investigated fiber-optic communications networks. From March 1988 to July 1993, he was at Bellcore (now Telcordia), Piscataway, NJ, where he engaged in broadband network research. Since 1993, he has been a Professor with the Department of Information Engineering, The Chinese University of Hong Kong, Hong Kong. $\mathrm{He}$ is an Adjunct Professor with the Southeast University, Nanjing, China. He is also currently a Consultant with the Hong Kong Applied Science and Technology Research Institute, Shatin, Hong Kong, providing technical advice as well as helping to formulate research and development directions and strategies in the areas of wireless internetworking, applications, and services. Separately, TCP Veno, which is a version of TCP that improves its performance over wireless networks, which was proposed by him and one of his students, has been incorporated into a recent release of Linux OS. In addition, in 1993, he initiated and built the first interuniversity asynchronous transfer mode network testbed in Hong Kong. More recently, his research group pioneered the concept of physical-layer network coding for application in wireless networks. Besides academic activities, he is also active in industry. $\mathrm{He}$ cofounded two technology startups in Internet software and has been serving as a consultant to many companies and industrial organizations. He is the holder of six U.S. patents. His current research interests include wireless networks, Internet protocols, multimedia communications, and packet switch design.

Dr. Liew is a Fellow of the Institution of Electrical Engineers and the Hong Kong Institution of Engineers. He is listed in Marquis Who's Who in Science and Engineering. He was the recipient of the first Vice-Chancellor Exemplary Teaching Award at The Chinese University of Hong Kong. He and one of his students won the Best Paper Awards at the 2004 IEEE International Conference on Mobile Ad-hoc and Sensor Systems and the 2004 IEEE International Workshop on Wireless Local Networks. 